\title{
Mesures et modélisations d'effets de saturation dans un photocommutateur picoseconde en GaAs BT
}

\author{
Hervé Eusèbe ${ }^{1}$, Jean-François Roux ${ }^{1}$, Frédéric Garet ${ }^{1}$, Jean-Louis Coutaz ${ }^{1}$ \\ Arunas Krotkus ${ }^{2}$, Klemensas Bertulis ${ }^{2}$ \\ ${ }^{1}$ LAHC, Université de Savoie, Campus Scientifique, 73376 Le Bourget du Lac, France \\ ${ }^{2}$ Semiconductor Physics Institute, 2600 A. Gostauto 11, Vilnius, Lituanie
}

\begin{abstract}
Résumé - Nous présentons ici des mesures et les modélisations correspondantes d'effets de saturation dans un photocommutateur picoseconde fabriqué en GaAs épitaxié à basse température et dopé avec du béryllium. Nous avons développé un modèle prenant en compte les aspects hyperfréquence et matériau. Ainsi nous pouvons observer et expliquer la limitation de l'amplitude des impulsions ainsi que l'augmentation de leur durée sous fort éclairement. Le modèle développé permet d'extraire certaines caractéristiques du dispositif et en particulier la densité de piège dans le matériau rapide.
\end{abstract}

Mots clés - LT GaAs, picoseconde, térahertz, photocommutation, saturation, pièges

\section{INTRODUCTION}

Les photocommutateurs (PC) à semi-conducteurs constituent actuellement les dispositifs optoélectriques les plus rapides, pouvant délivrer des impulsions électriques sub-picosecondes. Pour cela, on utilise des matériaux semi-conducteurs possédant une grande densité de défauts qui servent à piéger très efficacement les charges photogénérées. Ainsi, la durée de vie des électrons peut être inférieure à 100 fs [1] dans des matériaux non stœchiométriques comme GaAs épitaxié à basse température (LT-GaAs), où un excès d'arsenic forme des défauts de type antisite. Cependant, la réponse du détecteur n'est pas seulement déterminée par la réponse du matériau, mais aussi par celle du circuit hyperfréquences. Un éclairage à haute puissance du PC a deux effets associés sur la réponse du PC. Tout d'abord il peut saturer les niveaux de pièges dans le matériau, et ainsi allonger le temps de vie des porteurs et la réponse du PC. Ensuite il peut dépolariser le circuit électrique, générant alors une impulsion de plus faible amplitude que dans un cas linéaire. Dans cet article, nous mettons en évidence ces différents effets en appuyant les mesures par un modèle simple matériau/hyperfréquences.

\section{DISPOSITIF ETUDIE ET MODELE DEVELOPPE}

Le dispositif se présente sous la forme d'une ligne coplanaire en or déposée sur le semi- conducteur rapide. Le PC est situé au milieu du ruban central de la ligne (Fig. 1).

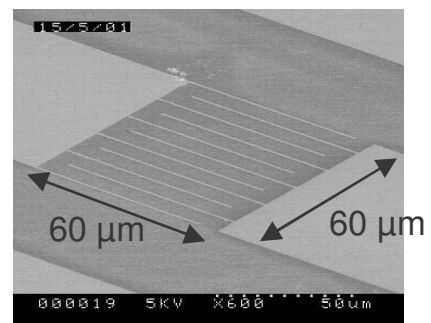

Fig.1 Photographie du PC

Le matériau est du LT-GaAs dopé avec du béryllium, épitaxié sur un substrat de GaAs [2]. Le béryllium permet de contrôler le pourcentage de défauts ionisés formant les pièges. Ce matériau se distingue par le temps de vie très court $(<0.5 \mathrm{ps})$ des photo-électrons, leur mobilitérelativement élevée $\left(500 \mathrm{~cm} \cdot \mathrm{V}^{-1} \cdot \mathrm{s}^{-1}\right)$ et une conductivité d'obscurité très faible. Le PC lui-même est constitué d'un peigne interdigité afin d'augmenter son efficacité. La largeur des doigts est de $1 \mu \mathrm{m}$ et leur espacement est d'environ $9 \mu \mathrm{m}$.

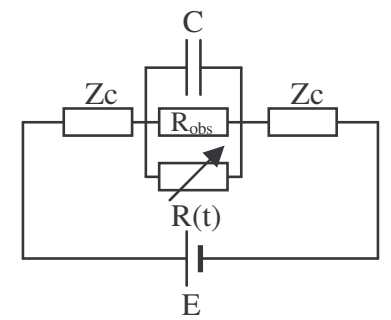

Fig.2 Modèle électrique du PC

Le modèle (Fig. 2). est basé sur un schéma électrique équivalent du PC. La valeur de la résistance variable est calculée en fonction de la densité de photoporteurs à chaque instant et de la géométrie du détecteur. La mobilité des porteurs est considérée comme constante et leurs durées de vie est calculée à partir la relation de Schokley Read et Hall (Equ. 1 avec $\mathrm{As}_{\mathrm{Ga}}{ }^{+}$la densité d'antisites ionisés (pièges libres), $\sigma_{n}$ la section efficace de capture et $v_{t h}$ la vitesse thermique moyenne des électrons). 


$$
\tau_{n}=\frac{1}{A s_{G a}{ }^{+} \cdot \sigma_{n} \cdot v_{t h}}
$$

La capacité est calculée par transformation conforme la structure interdigitée. Les lignes étant modélisées avec des résistances, la réponse calculée est celle au niveau des plans du détecteur. Le système est résolu numériquement par FDTD.

\section{Mesures ET DISCUSSION}

Dans un premier temps, nous effectuons une mesure par échantillonnage photo-conductif. Une première impulsion laser sur le $\mathrm{PC}$ génère une impulsion électrique qui se propage le long de la ligne coplanaire. Une deuxième impulsion laser, décalée temporellement de $\tau$, vient court-circuiter la ligne avec un ruban de masse et diminue l'amplitude du courant mesuré en bout de ligne. En faisant varier $\tau$, on reconstruit par échantillonnage la forme de l'impulsion électrique (Fig. 3). Nous observons un allongement de la durée des impulsions à haute puissance. Cette augmentation se produit brutalement pour des puissances supérieures à $16 \mathrm{~mW}$, valeur qui correspond à une densité de porteurs photo-générés égale à celle des pièges dans le matériau.

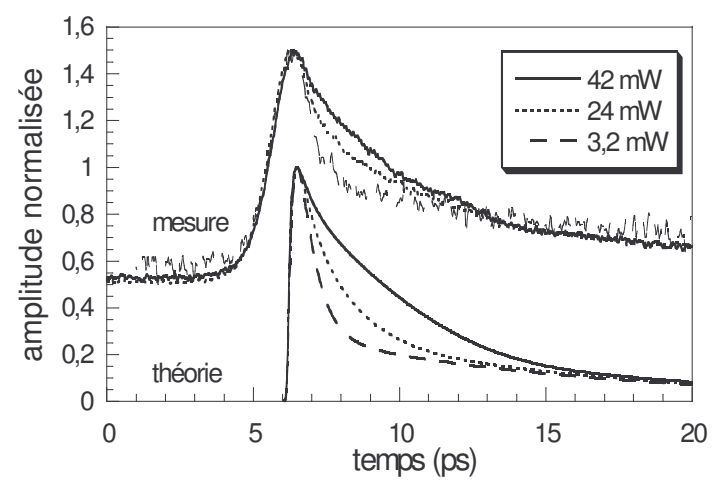

Fig.3 Augmentation de la durée de l'impulsion due à la saturation du matériau

Nous attribuons donc cet effet à une saturation des pièges. La densité d'électrons piégés devenant comparable à la densité de pièges libres à l'équilibre $\left(\mathrm{As}_{\mathrm{Ga}}{ }^{+}\right)$, leur durée de vie est alors déterminée par la recombinaison des électrons piégés qui met en jeu les trous. Dans l'exemple de la Fig. 3, la durée de décroissance du signal de corrélation passe de 1,65 ps à faible puissance à 5,5 ps en régime de saturation. Le modèle permet de retrouver ce comportement et de confirmer la densité d'antisites ionisés $\left(\left[A s_{G a}{ }^{+}\right]=5 \times 10^{17} \mathrm{~cm}^{-3}\right.$ [2])

La deuxième courbe (Fig. 4) représente l'amplitude des impulsions générées en fonction de la puissance optique éclairant le PC. La mesure est réalisée par échantillonnage électro-optique avec un cristal de tantalate de lithium posé sur les lignes. La mesure fait apparaître une saturation de l'amplitude pour des fortes puissances optiques.

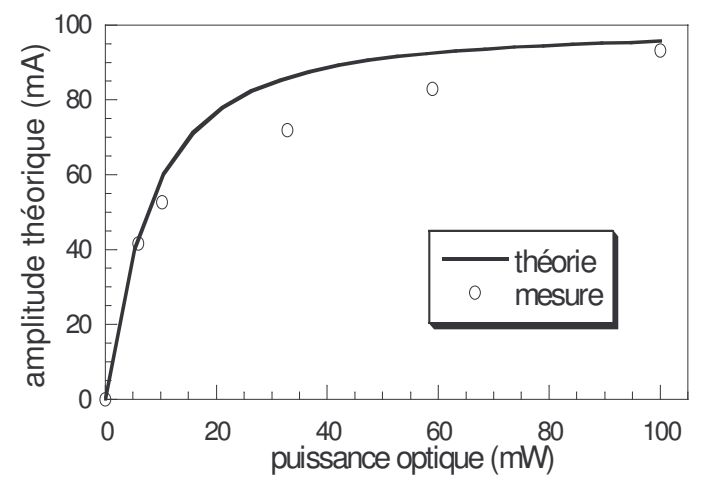

Fig.4 limitation de l'amplitude des impulsions par le circuit hyperfréquence

Ici nous voyons l'influence des lignes d'accès au détecteur. En effet, en se reportant au modèle électrique nous voyons que le courant maximum est limité par l'impédance des lignes à $E / 2 Z c$. L'impulsion générée dépolarise le PC et limite ainsi son amplitude. La saturation commence à apparaître lorsque la résistance minimum du PC devient comparable à l'impédance des lignes. Le modèle décrit très bien ce comportement.

\section{CONCLUSION}

Nous avons mesuré et modélisé la réponse d'un photocommutateur ultra-rapide. Ce travail a permis d'observer deux non-linéarités dues au circuit hyperfréquence et au matériau. Nous avons montré la saturation des pièges induisant une augmentation de la durée de l'impulsion photo générée par un à semi-conducteur de type III-V épitaxié à basse température. Le modèle théorique, basé sur un schéma électrique équivalent du PC, confirme nos hypothèses puisqu'il permet de retrouver l'allure des courbes expérimentales. De plus, nous avons pu extraire la densité d'antisites ionisés à l'équilibre. Enfin, nous avons observé et calculé la saturation de l'amplitude des impulsions sous forte puissance optique due à l'impédance du circuit hyperfréquence.

\section{REFERENCES}

[1] S. Marcinkevicius, A. Krotkus, R. Viselga, U. Olin and C. Jagadish, Semicond. Sci. Technol. 12, 396 (1997)

[2] A. Krotkus, K. Bertulis, M. Kaminska, K. Korona, A. Wolos, J. Siegert, S. Marcinkevicius, J.-F. Roux and J.-L. Coutaz, IEE Proc. - Optoelectr. 149, 111-115 (2002) 